\section{Differentiating Allele Combinations of a Transgene Using Multiple Primer PCR}

BioTechniques 20:956-960 (June 1996)

The production of a mouse line with a specific targeted gene interruption in both alleles requires the screening of embryonic stem (ES) cells and the genotyping of mouse genomic DNA. Typically the screening of ES cells colonies and tissue samples from transgenic mice is carried out using Southern blot analysis and/or the polymerase chain reaction (PCR). Southern blot analysis has the advantage of providing complete genotyping information on a single blot. However, Southern blots require the use of radioisotopes and they require substantially more DNA and take longer to perform than PCR. Conventional two-primer PCR genotyping is quicker and easier to perform than Southern blot analysis (5). Although the use of quantitative PCR techniques can distinguish between different alleles, they are complicated by the necessity of using exogenous (4) or endogenous (1) reference PCR control templates and quantitative scanning.

As an alternative to quantitative PCR, two PCRs using two different sets of primers (one primer set for the amplification of the junction fragment of a transgene and another set for the amplification of the wild-type gene fragment at the point of interruption) can be carried out. However, in addition to increasing the time and materials required, this approach can also give false-negative results because the absence of a PCR product is typically interpreted as an absence of the amplified sequence. However, the absence of a PCR product could also be caused by other factors such as those associated with the sample preparation. Here we outline a multiple primer PCR approach that combines the genotype information inherent in a Southern blot analysis with the speed and convenience of conventional two-primer PCR. Our procedure also eliminates the possibility of false-negative results in the genotyping of mouse or ES cell lines.

Several previous studies have utilized multiple primer PCR (1-3). However, in most cases, multiple primer PCR was carried out by using primers that amplify non-overlapping DNA fragments. A situation in which multiple PCR is carried out by using primers that simultaneously amplify overlapping fragments in the same reaction tube is more complex and has not been extensively investigated. An example of this situation is the genotyping of mutant mice when one wants to amplify the junction fragment of a transgene along with the wild-type gene fragment using one forward primer and two or more reverse primers. It is not clear which product or products would be produced by Taq DNA polymerase if one or more pair(s) of primers flank the overlapping fragments on the same DNA template. In a three-primer PCR experiment, there are three possibilities: 1) only the shorter fragment is amplified, 2) only the larger fragment is amplified or 3) both products are amplified. To ascertain which of these possibilities prevails in a multiple-primer PCR, we conducted experiments on a model system by using the DNA coding for mGluR4a, a G-protein-coupled metabotropic glutamate receptor.

The cDNA coding for mGluR4 in phagemid pBluescript ${ }^{\circledR} \mathrm{KS}(+)$ ) (Stratagene, La Jolla, CA, USA) was used as the template for PCR. Four primers, A, B, C and D (see Figure 1A), were included in the reaction mixtures in different combinations (see Figure 1B). Each reaction contained $50 \mathrm{ng}$ of pmGluR4, $2 \mu \mathrm{L}$ of $10 \times$ Taq DNA Polymerase buffer (Pharmacia Biotech, Uppsala, Sweden), $2 \mu \mathrm{L}$ of dNTP mixture (mixture of equal vol of $10 \mathrm{mM}$ solutions of dATP, dCTP, dGTP and dTTP), 40 pmol of each of the corresponding primers (see Figure 1B) and $2.5 \mu \mathrm{L}$ of Taq DNA Polymerase (5 U/ $\mu \mathrm{L}$ ) (Pharmacia Biotech). The volume of each reaction mixture was adjusted to $20 \mu \mathrm{L}$ with deionized water. The PCRs were carried out using a Programmable

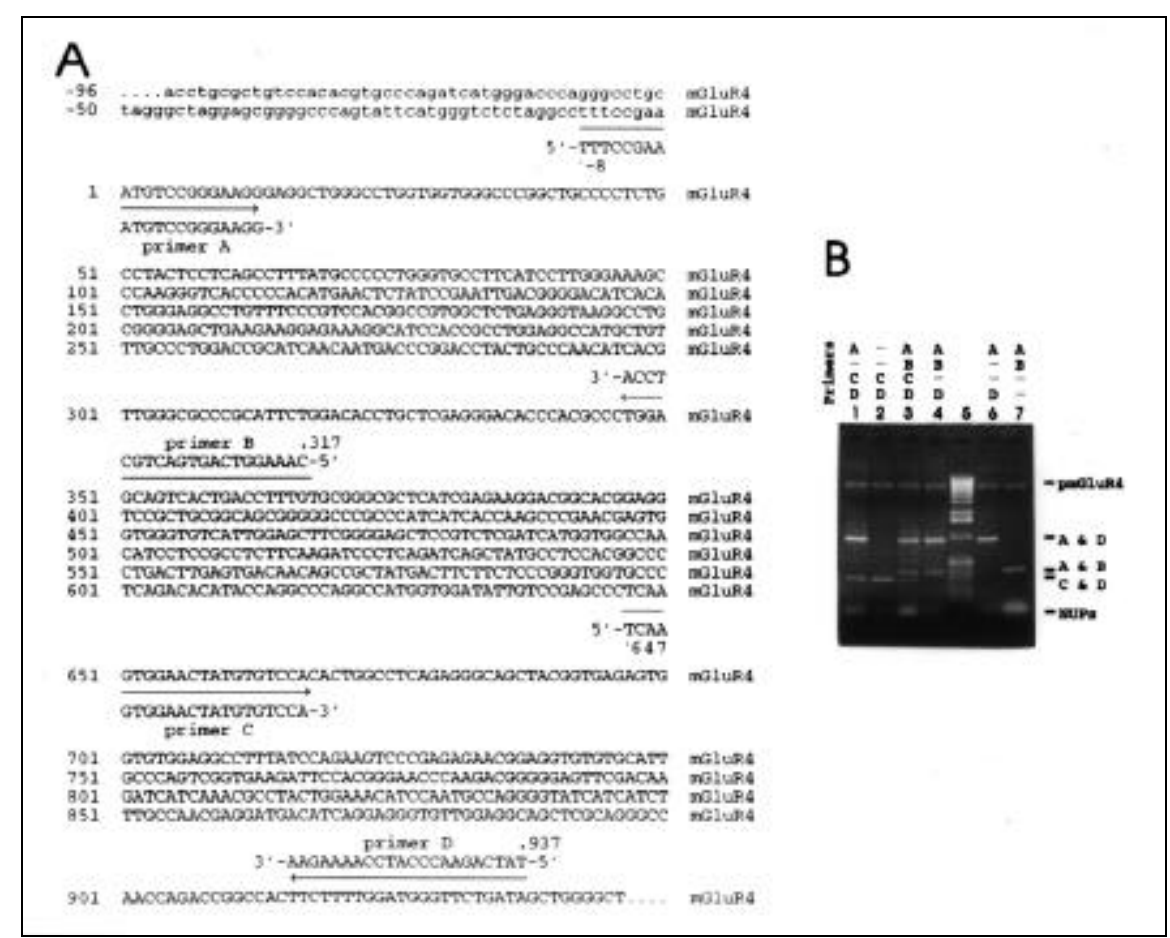

Figure 1. Multiple PCR of overlapping fragments of mGluR4a cDNA. (A) Position of the primers A, $\mathrm{B}, \mathrm{C}$ and $\mathrm{D}$ on the mGluR4a template cDNA. Only the relevant sequence of the mGluR4a cDNA (GenBank ${ }^{\circledR}$ Accession No. M90518) is represented. The $5^{\prime}$-untranslated region is shown in small letters. The numbers refer to the distance (bp) upstream (negative numbers) or downstream (positive numbers) from the first nucleotide of the translation start-codon. (B) The PCR products (lanes 1, 2, 3, 4, 6 and 7). The primers used are indicated above each lane number. Lane 5 is a 1-kb Ladder (Life Technologies, Burlington, ON, Canada). NUPr are non-utilized primers. pmGluR4 indicates the position of the phagemid, which was used as a template for the PCRs (see text). 
Thermal Controller (PTC-100 ${ }^{\mathrm{TM}}$, Model 60; MJ Research, Watertown, MA, USA) under the following conditions: $30 \mathrm{~s}$ at $90^{\circ} \mathrm{C}, 1 \mathrm{~min}$ at $56^{\circ} \mathrm{C} ; 2 \mathrm{~min}$ at $72^{\circ} \mathrm{C}$ for 7 cycles. After amplification, a $15-\mu \mathrm{L}$ aliquot from each reaction was separated on a $1 \%$ agarose gel at $75 \mathrm{~V}$ for $1 \mathrm{~h}$ and visualized by ethidium bromide staining.

Although we used four different primers in this study, all possible primer combinations could theoretically produce only three PCR products of 325,945 and $290 \mathrm{bp}$ corresponding to primers combinations of A-B, A-D and $\mathrm{C}-\mathrm{D}$, respectively. As seen in Figure $1 \mathrm{~B}$, the corresponding PCR products were amplified using conventional two-primer PCR (lanes 2, 6 and 7), and using three-primer (lanes 1 and 4) and four-primer (lane 3) PCR when corresponding primer pairs were used. Based on these results, we conclude that Taq DNA polymerase is capable of producing at least three PCR products, even if these products originated from overlapping fragments of the same DNA template when three or four primers were used simultaneously.

In a second experiment, we sought to demonstrate the applicability of three-primer PCR to the genotyping of transgenic mice. In order to apply three-primer multiple PCR for distinguishing homozygous, heterozygous and wild-type mice in the same reaction, two of the primers should be designed to amplify the junction fragment of a transgene. These two primers should consist of an allele-nonspecific

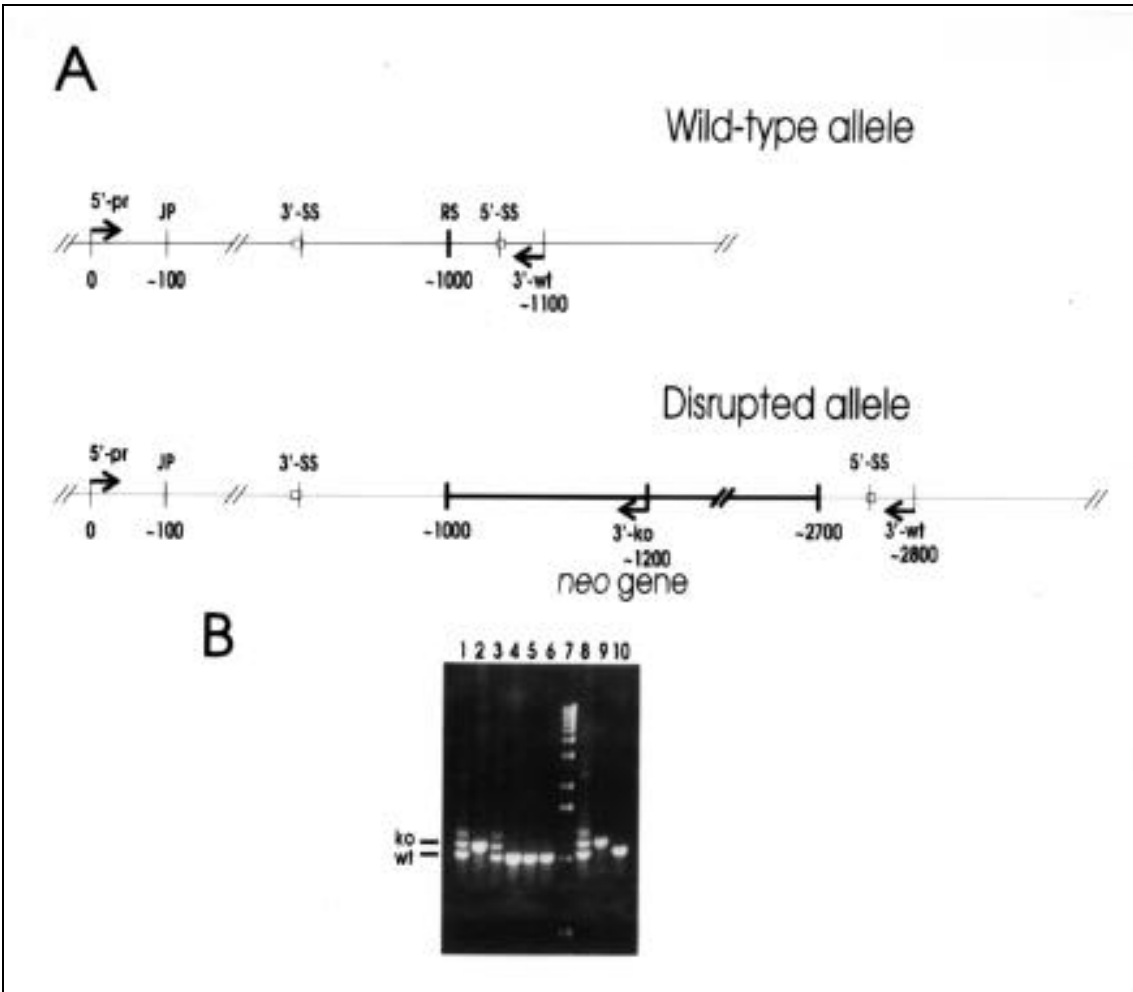

Figure 2. The use of three-primer multiple PCR for genotyping transgenic mice. Panel A: Positions of $5^{\prime}$-primer (5'-pr), $3^{\prime}$-wild-type specific primer (3'-wt) and $3^{\prime}$-knock-out specific primer (3'-ko) on wild-type and disrupted alleles of a hypothetical gene used in a typical targeted gene disruption experiment. Arrows represent primers and the boxes show $3^{\prime}$ - and $5^{\prime}$-splicing sites ( $3^{\prime}$-ss and $5^{\prime}$-ss, respectively). The exact cutting points within the splicing sites and the points of annealing of the $5^{\prime}$-end of each primer are represented by vertical lines. The numbers underneath refer to the approximate distance (bp) downstream from the annealing point of the $5^{\prime}$-end of the $5^{\prime}$-pr primer. The sequence of genomic DNA is represented by a thin line, and the neo cDNA expression cassette is represented by a thick line. The cutting point of a restriction site (RS), utilized to disrupt the hypothetical protein-coding sequence, is shown by a thick vertical line. The $5^{\prime}$-border of the gene fragment included in the targeting vector is indicated by JP (junction point). Panel B: Genotyping of mGluR4 mutant mice. Lanes 1-6 and 8-10 are mouse genomic DNA, lane 7 is a $1-\mathrm{kb}$ Ladder (Life Technologies), wt indicates the position of the wild-type allele-specific band (1068 bp) and ko indicates the disrupted allele-specific band (1170 bp). primer (i.e., a primer common to both alleles) and a transgene-specific primer (see Figure 2A; 5'-primer and 3'knock-out primer, respectively). The third primer should be designed such that, when it is used in conjunction with the allele-nonspecific primer (5'pr primer), the amplified product will be similar in size to the junction fragment, but electrophoretically distinguishable from it (Figure 2A, 3'-wildtype primer).

The sequences of the three primers that we used for genotyping mmGluR4 knock-out mice were: $\mathrm{m} 4-5^{\prime}$-pr - 5'-atgcagaggtgtctcatacctg- $3^{\prime}$; neo- $3^{\prime}$-ko - 5'ggtggatgtggaatgtgtgcg- $3^{\prime}$; and $\mathrm{m} 4-3^{\prime}-$ wt - 5'-gcctgggctactgtctgtcat- $3^{\prime}$. Threeprimer multiple PCRs were carried out with 1-2 $\mu \mathrm{g}$ of genomic DNA, $250 \mu \mathrm{M}$ of each dNTP, 40 pmol of each primer (m4-5'-pr, m4-3'-wt and 3'-ko), $1 \times$ Taq DNA Polymerase buffer, Milli-Q ${ }^{\circledR}$ water (Millipore, Bedford, MA, USA) (to a final volume of $50 \mu \mathrm{L}$ ) and $1.5 \mathrm{U}$ of Taq DNA Polymerase (Pharmacia Biotech). PCR conditions were as follows: $40 \mathrm{~s}$ at $95^{\circ} \mathrm{C}, 30 \mathrm{~s}$ at $62^{\circ} \mathrm{C}$ and 3 $\min$ at $72^{\circ} \mathrm{C}$ for 32 cycles. This was followed by additional incubation at $72^{\circ} \mathrm{C}$ for $5 \mathrm{~min}$. Twenty microliters of each amplification mixture were separated on a $1 \%$ agarose gel at $75 \mathrm{~V}$ for $1.5 \mathrm{~h}$.

In an hypothetical example shown schematically in Figure 2A, three products could theoretically be produced by PCR utilizing a 5'-pr, a 3'-ko and a $3^{\prime}$-wt primer when heterozygous genomic DNA is used as a template. These include approximately a 1000-bp product of the $5^{\prime}$-pr and $3^{\prime}$-wt primers from the wild-type allele, approximately a 1100-bp product from the $5^{\prime}$-pr-3'-ko primers from the disrupted mutant allele and approximately a 2800 -bp product from the $5^{\prime}$-pr- $3^{\prime}$-wt primers also from the mutant allele. However, because of its length, the largest fragment would not be expected to be amplified sufficiently for visualization by staining with ethidium bromide. In the genotyping of the mGluR4 mutant mice, the corresponding largest band would be 2838 bp in length. This 2838 bp band was not seen, even when $2 \mu \mathrm{g}$ of plasmid DNA were used as the template in conventional two-primer PCR (data not shown). The 2838-bp product was also not visible in mGluR4 mutant 


\section{Benchmarks}

mouse genotyping by the three-primer multiple PCR. However, both of the smaller PCR products of 1068 (specific for the wild-type allele of mGluR4) and 1170 bp (specific for the mGluR4 transgene) were visible. Thus, all three allele combinations were clearly distinguishable on agarose gels stained with ethidium bromide. DNA from wildtype mice give a single smaller band (1068 bp; see Figure 2B, lanes 4, 5, 6 and 10). All transgenic homozygous mice showed the 1170-bp product (Figure 2B, lanes 2 and 9), and the heterozygous mice showed both PCR products (Figure 2B, lanes 1, 3 and 8). The results of the three-primer multiple PCR genotyping of mGluR4 mutant mice were confirmed by Southern blot and immunoblot analysis (data not shown).

The results presented here confirm that multiple primer PCR can be applied to the genotyping of transgenic mice. The three-primer protocol outlined above increases the reliability of a genotyping screen because, unlike conventional two-primer PCR, threeprimer PCR will give a PCR product regardless of the genotype. Our results indicate that three-primer multiple PCR provides a very useful alternative for genotyping transgenic mouse and ES cells lines.

\section{REFERENCES}

1.Chatelain, G., G. Brun and D. Michel. 1995. Screening of homozygous transgenic mice by comparative PCR. BioTechniques 18:958962.

2.Padegimas, L., O.A. Shul'ga and K.G. Skriabin. 1993. Testing of transgenic plants using the polymerase chain reaction. Mol. Biol. 27:947-951

3.Peng, H.Z., P.G. Isaacson, T.C. Diss and L.X. Pan. 1994. Multiple PCR analyses on trace amounts of DNA extracted from fresh and paraffin wax embedded tissues after random hexamer primer PCR amplification. J. Clin. Pathol. 47:605-608.

4.Siebert, P.D. and J.W. Larrick. 1993. PCR MIMICS: competitive DNA fragments for use as internal standards in quantitative PCR. BioTechniques 14:244-249.

5.Ward, M., W. Abramow-Newerly, B. Clapoff and J.C. Roder. 1992. Analysis of transgenic animal pedigrees using the junction-polymerase chain reaction. PCR Methods Appl. 1:208-209.

This work was supported by the Medical Research Council (Canada) Group of Nerve
Cells and Synapses. Address correspondence to David R. Hampson, Faculty of Pharmacy, University of Toronto, 19 Russell St., Toronto, Ontario, Canada M5S 2 S2. Internet: hampson@phm.utoronto.ca

Received 29 September 1995; accepted 13 December 1995.

\section{Roman Pekhletski and David R. Hampson \\ University of Toronto \\ Toronto, ON, Canada}

\section{An In Vitro Assay of $\beta$-Galactosidase from Yeast}

BioTechniques 20:960-962 (June 1996)

The quantification of $\beta$-galactosidase in yeast cells harboring a lac $\mathrm{Z}$ reporter gene linked to the promoter gene of interest is an important and commonly used procedure in many laboratories working with yeast cells. In particular, with the burgeoning use of interaction cloning strategies as typified by the yeast two-hybrid system, this assay has become indispensable for the accurate characterization of truly interacting clones and for subsequently determining their interaction domains.

In general, two methods are commonly used to lyse the yeast cells of interest prior to performing a $\beta$-galactosidase quantification reaction (8). One method (7) relies on the shearing and crude lysis of yeast cells induced by sequential rounds of vortex mixing of the cells with glass beads. Activity is then subsequently normalized by the amount of protein assayed. The second method (5) utilizes a permeabilization step in which the yeast cells are first rendered permeable to the enzymatic substrate and the activity is subsequently normalized by an estimate of the number of yeast cells assayed.

We have used both methods extensively and have found that for the accurate comparison of $\beta$-galactosidase activity from yeast two-hybrid strains such as Y153 (3) or RH6IIE (4) having different genetic backgrounds, the first method gives the most consistent and reproducible results, thus permitting an accurate ranking of the relative strengths of interaction among different clones.

While lysis of cells by glass bead vortex mixing is the preferred method, it has a drawback of being a very tedious and time-consuming procedure, particularly when larger numbers of samples have to be analyzed.

We therefore sought an alternative procedure that would result in an effective and rapid lysis of the yeast cell wall and that would still permit an accurate quantification of $\beta$-galactosidase activity. 